SUSSEX-AST 95/1-1

astro-ph/9501086

(January 1995)

\title{
Generalised Scalar Field Potentials and Inflation
}

\author{
Paul Parsons and John D. Barrow \\ Astronomy Centre, \\ School of Mathematical and Physical Sciences, \\ University of Sussex, \\ Brighton BN1 9QH, U. K.
}

\begin{abstract}
We investigate the range of inflationary universe models driven by scalar fields possessing a general interaction potential of the form $V(\phi)=V_{0} \phi^{n} \exp \left(-\lambda \phi^{m}\right)$. Power-law, de Sitter and intermediate inflationary universes emerge as special cases together with several new varieties of inflation. Analysing the behaviour of these models at the extrema of $\phi$ we derive sufficient constraints on the $m-n$ parameter space such that inflation may occur as both an early and late-time phenomenon. We also compute the scalar and tensor perturbations produced in the model and compare these results with recent observations.
\end{abstract}

PACS number 98.80.Cq 


\section{Introduction}

Inflationary cosmologies have become the most popular models of the early universe. Their ability to solve many of the problems inherent in the standard cosmology [1, 2], whilst simultaneously offering a mechanism for the generation of the seed fluctuations for structure formation [3], consistent with recent observations [4, 5], make inflation a very elegant and appealing scenario. Many variations now exist: in addition to Guth's original version with an ephemeral cosmological constant [6], there exist models in which the self-interaction potential $V$ for the inflaton field $\phi$ assumes exponential [0, 8, 9] or positive power-law forms [10]. These potentials give rise to power-law and polynomial-chaotic inflation respectively [10, 11. Also of current interest, because of their ability to produce unusual perturbation spectra, are the intermediate inflationary models of $[12-15]$. These arise when the potential behaves asymptotically as a decaying power-law.

In this paper we endeavour to combine all of these models as special cases of a single more general potential,

$$
V(\phi)=V_{0} \phi^{n} \exp \left(-\lambda \phi^{m}\right)
$$

This possibility was originally examined by Barrow [16], where it was shown that $m \leq 1$ was a necessary condition for inflation at large values of $\phi$. In the absence of an exact solution for the potential of Eq. (11.1) we demonstrate that the slow-roll approximation [17] is generically satisfied at large $\phi$ in all such models, when $m \leq 1$. We employ asymptotic techniques to ascertain the behaviour of the system at large $\phi$ in all regions of the $m-n$

parameter space, revealing some new types of inflationary universe. We are able to verify that $m \leq 1$ is also a sufficient condition for the potential to support an epoch of inflation at large $\phi$, and there are no constraints on the value of $n$ in this limit. We find that when $0<m \leq 1$ inflation occurs as a late-time phenomenon. However, in most cases with $m<0$ 
we find that $\phi$ grows large as $t$ becomes small and inflation is an early time feature. In these models $\dot{\phi}$ is negative and the potential steepens at small $\phi$ to end inflation naturally. Furthermore, if $m<0$, and $V$ is an even function of $\phi$, then the potential will contain a minimum at $\phi=0$, which allows the field ultimately to decay into particles that reheat the universe once inflation ends 11, without requiring additional modifications to the model. We also find that when $m>1$, inflation occurs uniquely at early times before the kinetic energy of the field comes to dominate as $t \rightarrow \infty$. If $0<m<1$ inflation proceeds indefinitely. This also occurs in some models with $m<0$ and should be viewed as describing the behaviour of the system on a discrete portion of some grander potential with a minimum. Finally, we

calculate the scalar and tensor fluctuation spectra arising in the generalised model. We use these results in conjunction with observations to constrain further the allowed parameter values in Eq. (1.1).

\section{Equations of Motion}

We shall concern ourselves with the behaviour of a zero-curvature Friedmann universe. The matter content is dominated by a homogeneous scalar field $\phi$ with potential $V(\phi)$. The scale factor of the universe is $a(t)$, where $t$ is synchronous cosmic time, and we define the Hubble expansion parameter as

$$
H=\frac{\dot{a}}{a},
$$

where an overdot represents a derivative with respect to $t$. Einstein's equations (in units with $\left.8 \pi M_{P l}^{-2}=c=1\right)$ are then

$$
\begin{aligned}
\ddot{\phi}+3 H \dot{\phi} & =-V^{\prime}, \\
3 H^{2} & =\frac{1}{2} \dot{\phi}^{2}+V, \\
\dot{H} & =-\frac{1}{2} \dot{\phi}^{2},
\end{aligned}
$$


where primes denote derivatives with respect to the scalar field and $M_{P l}$ is the Planck mass. Solution of these equations exactly is not always possible, although we can often make progress within the context of the slow-roll approximation. The Hubble Slow-Roll Approximation (HSRA) [17] is defined by the smallness of a set of parameters, the first two of which are given by

$$
\begin{aligned}
& \epsilon_{H}=2\left(\frac{H^{\prime}}{H}\right)^{2}\left(\equiv 3 \frac{\dot{\phi}^{2} / 2}{V+\dot{\phi}^{2} / 2}\right), \\
& \eta_{H}=2 \frac{H^{\prime \prime}}{H}\left(\equiv-3 \frac{\ddot{\phi}}{3 H \dot{\phi}}\right) .
\end{aligned}
$$

The condition $\epsilon_{H}<1$ is identical to the condition $\ddot{a}(t)>0$ for inflation. In 17] the HSRA was formulated as a perturbative expansion, here we shall just be concerned with the zeroth order approximation, which neglects the kinetic terms $\left(\dot{\phi}^{2}\right.$ and $\left.\ddot{\phi}\right)$ in the equations of motion. In this regime Eqs. (2.2) - (2.3) simplify to

$$
\begin{aligned}
& 3 H^{2}=V, \\
& 3 H \dot{\phi}=-V^{\prime} .
\end{aligned}
$$

Hence,

$$
\dot{\phi}=-\frac{V^{\prime}}{\sqrt{3 V}} .
$$

For completeness we also mention the Potential Slow-Roll Approximation (PSRA) [17, the validity of which is given by the smallness of the parameters

$$
\begin{aligned}
\epsilon_{V} & =\frac{1}{2}\left(\frac{V^{\prime}}{V}\right)^{2} \\
\eta_{V} & =\frac{V^{\prime \prime}}{V}
\end{aligned}
$$

The PSRA is in some respects more useful than the HSRA in that it allows a certain amount of insight into the behaviour of the system simply from examining the form of 
the potential. However, one should exercise caution with such an approach. Smallness of the PSRA parameters only classifies the flatness of the potential; it carries no information regarding the initial conditions $\left\{\phi_{0}, \dot{\phi}_{0}\right\}$ and so offers no guarantee that the field will be genuinely slow-rolling. Thus for the PSRA to be valid requires the additional assumption that the evolution has reached a form of attractor solution, onto which all trajectories from all possible initial conditions will have converged to within acceptable tolerances. This is the inflationary attractor hypothesis discussed in [17]. The parameters $\epsilon_{V}$ and $\eta_{V}$ for the potential of Eq. (1.1) are

$$
\begin{aligned}
\epsilon_{V} & =\frac{1}{2 \phi^{2}}\left(n-\lambda m \phi^{m}\right)^{2} \\
\eta_{V} & =\frac{1}{\phi^{2}}\left[n\left(\frac{n}{2}-1\right)-\lambda m(n+m-1) \phi^{m}+\frac{\lambda^{2} m^{2}}{2} \phi^{2 m}\right] .
\end{aligned}
$$

These expressions will prove useful in our later discussion of perturbation spectra as well as providing a reliable probe for determining when inflation occurs.

\section{$3 \quad$ Asymptotic Analysis of $\mathbf{V}(\phi)=\mathbf{V}_{\mathbf{0}} \phi^{\mathbf{n}} \exp \left(-\lambda \phi^{\mathbf{m}}\right)$}

The behaviour of inflationary universe models driven by potentials of the form, $V(\phi)=$ $V_{0} \phi^{n} \exp \left(-\lambda \phi^{m}\right)$, was first investigated and classified by Barrow [16], where it was shown that late-time inflation only occurs for models in which $m \leq 1$, encompassing a broad spectrum of possibilities. When $m=0$ and $n$ is positive we have polynomial-chaotic inflation, for negative $n$ the evolution asymptotes to intermediate inflation and when $m=1, n=0$ we retrieve the power-law inflationary solution. The choice $m=n=0$ leads back to Guth's original model [6] with exponential expansion.

We shall now examine how this model behaves in the parameter ranges $0<m<1$ and $m<0$. In these cases the equations of motion are not exactly soluble, although we may 
make progress within the context of the slow-roll approximation. Generically this will apply on the asymptotic region of the potential, ie at large $\phi$, allowing us to employ asymptotic techniques [18] to solve Eqs. (2.9) and (1.1) and obtain the complete slow-rolling solution.

\subsection{The case $0<\mathrm{m}<1$}

Differentiating Eq. (1.1) with respect to $\phi$ yields

$$
V^{\prime}=V_{o}\left(n-\lambda m \phi^{m}\right) \phi^{n-1} \exp \left(-\lambda \phi^{m}\right)
$$

We are working asymptotically in $\phi$, where the slow-roll conditions are well satisfied and Eqs. (2.7) - (2.9) are valid. Substituting Eq. (3.1) into Eq. (2.9) and taking the asymptotic limit gives

$$
\dot{\phi}=\sqrt{\frac{V_{o}}{3}} \lambda m \phi^{m+\frac{n}{2}-1} \exp \left(-\frac{\lambda}{2} \phi^{m}\right) .
$$

At large $\phi$ this expression integrates approximately to yield

$$
t(\phi)=\frac{2}{\lambda^{2} m^{2}} \sqrt{\frac{3}{V_{0}}} \phi^{2-2 m-\frac{n}{2}} \exp \left(\frac{\lambda}{2} \phi^{m}\right) .
$$

In the absence of an exact inversion to $\phi(t)$ we express Eq. (3.3) as,

$$
\left(2-2 m-\frac{n}{2}\right) \ln \phi+\frac{\lambda}{2} \phi^{m}=\ln \left[\sqrt{\frac{V_{0}}{3}} \frac{\lambda^{2} m^{2}}{2} t\right] .
$$

Asymptotically the power law in $\phi$ dominates the left hand side and so the lowest-order approximation is

$$
\phi(t)=\left(\frac{2}{\lambda}\right)^{\frac{1}{m}} \ln \frac{1}{m}\left[\sqrt{\frac{V_{0}}{3}} \frac{\lambda^{2} m^{2}}{2} t\right] .
$$

Rearranging Eq. (3.3) and substituting Eq. (3.5) yields $\phi(t)$ to second order,

$$
\phi(t)=\left(\frac{2}{\lambda}\right)^{\frac{1}{m}} \ln \frac{1}{m}\left[\frac{\sqrt{\frac{V_{0}}{3}} \frac{\lambda^{2} m^{2}}{2} t}{\left(\frac{2}{\lambda}\right)^{\frac{2}{m}-\frac{n}{2 m}-2} \ln \frac{2}{m}-\frac{n}{2 m}-2\left[\sqrt{\frac{V_{0}}{3}} \frac{\lambda^{2} m^{2}}{2} t\right]}\right] .
$$


We can now compute $H(t)$. From Eq. (2.7) we have

$$
H(\phi)=\sqrt{\frac{V_{o}}{3}} \phi^{\frac{n}{2}} \exp \left(-\frac{\lambda}{2} \phi^{m}\right) .
$$

Inserting Eq. (3.6) into Eq. (3.7) and taking the large $t$ limit $(t \rightarrow \infty$ as $\phi \rightarrow \infty)$, we find

$$
H(t)=\frac{1}{\lambda m^{2}}\left(\frac{2}{\lambda}\right)^{\frac{2-m}{m}} \frac{1}{t} \ln \frac{2-2 m}{m}\left[\sqrt{\frac{V_{o}}{3}} \frac{\lambda^{2} m^{2}}{2} t\right] .
$$

This expression may be integrated to obtain the behaviour of the scale factor. We thus obtain the full asymptotic time evolution of the system,

$$
\begin{aligned}
\phi(t) & =\left(\frac{2}{\lambda}\right)^{\frac{1}{m}} \ln ^{\frac{1}{m}} t, \\
H(t) & =\frac{1}{\lambda m^{2}}\left(\frac{2}{\lambda}\right)^{\frac{2-m}{m}} \frac{1}{t} \ln \frac{2-2 m}{m} t \\
a(t) & \propto \exp \left[\frac{1}{\lambda m(2-m)}\left(\frac{2}{\lambda}\right)^{\frac{2-m}{m}} \ln \frac{2-m}{m} t\right] .
\end{aligned}
$$

Analysing the positivity of $\ddot{a}(t)$ at large $t$ in the neighbourhood of $m=1$ confirms the result that inflation only occurs when $m \leq 1$. Thus in general we have a new form of inflation when $0<m<1, m \neq 1$. As might be expected, the solution reduces to power-law inflation when $m=1$ and is independent of $n$ to leading order.

\subsection{The case $\mathrm{m}<0$}

The behaviour of $\dot{\phi}$ at large $\phi$ for $m<0$ leads to three distinct cases:

\subsection{1 $m<0, n \neq 0,4$}

In this subclass of solutions the first term in Eq. (3.1) will dominate. Eqs. (2.7) and (2.8) then imply

$$
\dot{\phi}=-\sqrt{\frac{V_{o}}{3}} n \phi^{\frac{n}{2}-1} \exp \left(-\frac{\lambda}{2} \phi^{m}\right)
$$


At large $\phi$ the integral of this expression is well approximated by

$$
t(\phi)=\frac{1}{n} \sqrt{\frac{3}{V_{0}}}\left(\frac{2}{n-4}\right) \phi^{\frac{4-n}{2}} \exp \left(\frac{\lambda}{2} \phi^{m}\right),
$$

which may be inverted asymptotically. Substituting this result into Eq. (3.7) allows $H(t)$ to be approximated at large $\phi$, and integrated asymptotically to obtain $a(t)$. The full solution is

$$
\begin{aligned}
& \phi(t)=\left[\frac{n}{2} \sqrt{\frac{V_{0}}{3}}(n-4) t\right]^{\frac{2}{4-n}}, \\
& H(t)=\sqrt{\frac{V_{0}}{3}}\left[\frac{n}{2} \sqrt{\frac{V_{0}}{3}}(n-4)\right]^{\frac{n}{4-n}} t^{\frac{n}{4-n}} \exp \left[-\frac{\lambda}{2}\left[\frac{n}{2} \sqrt{\frac{V_{0}}{3}}\right]^{\frac{2 m}{4-n}} t^{\frac{2 m}{4-n}}\right], \\
& a(t) \propto \exp \left[\sqrt{\frac{V_{0}}{3}}\left(\frac{4-n}{4}\right)\left[\frac{n}{2}(n-4) \sqrt{\frac{V_{0}}{3}}\right]^{\frac{n}{4-n}} t^{\frac{4}{4-n}} \gamma_{1}(t)\right] ;
\end{aligned}
$$

where $\gamma_{1}(t)$ is given by

$$
\gamma_{1}(t) \equiv \exp \left\{-\frac{\lambda}{2}\left[\frac{n}{2}(n-4) \sqrt{\frac{V_{0}}{3}}\right]^{\frac{2 m}{4-n}} t^{\frac{2 m}{4-n}}\right\}
$$

and we note that $\gamma_{1}(t) \rightarrow 1$ as $\phi \rightarrow \infty$. Also,

$$
H(t) \rightarrow \sqrt{\frac{V_{0}}{3}}\left[\frac{n}{2}(n-4) \sqrt{\frac{V_{0}}{3}}\right] t^{\frac{n}{4-n}}
$$

for all $n$ as $\phi \rightarrow \infty$. Writing

$$
a(t) \propto \exp \left[\alpha t^{\beta} \gamma_{1}(t)\right]
$$

we find three subclasses, exhibiting the following asymptotic behaviour:

(i) $\mathbf{n}>4$. This implies $n(n-4)>0$ and $\alpha, \beta>0$. Eq. (3.13) reveals that $t \rightarrow 0$ as $\phi \rightarrow \infty$ - our asymptotic analysis is thus concerned with the earliest stages of the universe's evolution. 
(ii) $\mathbf{0}<\mathbf{n}<4$. Since $n(n-4)<0$, the constraints on Eq. (3.18) in this case are $\alpha>0,1<\beta<\infty$. Eq. (3.13) implies $t \rightarrow-\infty$ as $\phi \rightarrow \infty$ and inflation arises once again as a primordial effect ${ }^{1}$.

(iii) $\mathbf{n}<\mathbf{0}$. Here $n(n-4)>0$. The parameter constraints are $\alpha>0,0<\beta<1$. In this case we are concerned with the late-time evolution of the solution since from Eq. (3.13) $t \rightarrow \infty$ as $\phi \rightarrow \infty$. The solution asymptotes to an intermediate inflationary model.

Cases $(i)$ and (ii) display inflationary behaviour as an early-time feature in a manner akin to chaotic inflation models with concave potentials. Inflation proceeds for a finite time before switching off as the potential becomes too steep.

\subsection{2 $\mathrm{m}<0, \mathrm{n}=\mathbf{0}$}

Eqs. (2.7) and (2.8) imply

$$
\dot{\phi}=\sqrt{\frac{V_{0}}{3}} \lambda m \phi^{m-1} \exp \left(-\frac{\lambda}{2} \phi^{m}\right) .
$$

In the asymptotic limit $t(\phi)$ becomes

$$
t(\phi)=\sqrt{\frac{3}{V_{0}}} \frac{\phi^{2-m}}{\lambda m(2-m)} \exp \left(\frac{\lambda}{2} \phi^{m}\right) .
$$

Following the procedures of the previous section, we obtain the full asymptotic solution,

$$
\begin{aligned}
\phi(t) & =\left[\lambda m(2-m) \sqrt{\frac{V_{0}}{3}}\right]^{\frac{1}{2-m}} t^{\frac{1}{2-m}}, \\
H(t) & =\sqrt{\frac{V_{0}}{3}} \exp \left[-\frac{\lambda}{2}\left\{\lambda m(2-m) \sqrt{\frac{V_{0}}{3}}\right\}^{\frac{m}{2-m}} t^{\frac{m}{2-m}}\right],
\end{aligned}
$$

\footnotetext{
${ }^{1}$ The apparent discrepancy in the definition of early between cases $(i)$ and $(i i)(t \rightarrow 0$ and $t \rightarrow-\infty)$ is a result of the special choices of time co-ordinate arising in the particular solutions as a result of ignoring integration constants. Both should be taken to represent the early-time limit.
} 


$$
a(t) \propto \exp \left[\sqrt{\frac{V_{0}}{3}} t \gamma_{2}(t)\right]
$$

where,

$$
\gamma_{2}(t) \equiv \exp \left[-\frac{\lambda}{2}\left\{\lambda m(2-m) \sqrt{\frac{V_{0}}{3}}\right\}^{\frac{m}{2-m}} t^{\frac{m}{2-m}}\right]
$$

We see that $\gamma_{2}(t) \rightarrow 1$ as $\phi \rightarrow \infty$ and so we recover the de Sitter solution in this limit, as expected. We also see from Eq. (3.20) that $t \rightarrow-\infty$ as $\phi \rightarrow \infty$ and inflation is an early-time feature in the evolution of the universe.

\subsection{3 $\mathrm{m}<0, \mathrm{n}=4$}

The behaviour of $\dot{\phi}$ at large $\phi$ for this model is identical to the case for arbitrary $n$. We have

$$
\dot{\phi}=-4 \sqrt{\frac{V_{0}}{3}} \phi \exp \left(-\frac{\lambda}{2} \phi^{m}\right) .
$$

However, when this function is integrated in the asymptotic limit we encounter qualitatively different behaviour, vested in the appearance of a logarithmic factor,

$$
t(\phi)=-\frac{1}{4} \sqrt{\frac{3}{V_{0}}}(\ln \phi) \exp \left(\frac{\lambda}{2} \phi^{m}\right) .
$$

Consequently, the asymptotic solution differs substantially,

$$
\begin{aligned}
\phi(t) & =\exp \left[-4 \sqrt{\frac{V_{0}}{3}} t\right], \\
H(t) & =\sqrt{\frac{V_{0}}{3}} \exp \left[-8\left(\frac{V_{0}}{3}\right)^{\frac{1}{2}} t-\frac{\lambda}{2} \exp \left\{-4 m \sqrt{\frac{V_{0}}{3}} t\right\}\right], \\
a(t) & \propto \exp \left\{-\frac{1}{8} \exp \left[-8 \sqrt{\frac{V_{0}}{3}} t\right] \gamma_{3}(t)\right\},
\end{aligned}
$$

where $\gamma_{3}(t)$ here takes the form

$$
\gamma_{3}(t) \equiv \exp \left[-\frac{\lambda}{2} \exp \left\{-4 m\left(\frac{V_{0}}{3}\right)^{\frac{1}{2}} t\right\}\right]
$$


and tends to unity in the large $\phi$ limit. Eq. (3.27) reveals that $t \rightarrow-\infty$ when $\phi \rightarrow \infty$; once again we are dealing with the early evolution of the system and Eq. (3.29) verifies the asymptotic approach of the model to standard $V(\phi) \propto \phi^{4}$ models. Potentials such as this, which are symmetric about $\phi=0$, contain minima and allow a natural end to inflation within the model. One can thus expect a finite amount of inflation in these theories, dependent on the initial value of $\phi$.

\section{Analysis of $\mathbf{V}(\phi)=\mathbf{V}_{\mathbf{0}} \phi^{\mathbf{n}} \exp \left(-\lambda \phi^{\mathbf{m}}\right)$ at small $\phi$}

In many of the models examined in the previous section, inflationary behaviour arose as an early-time feature at large $\phi$ values. This poses the question of inflation at small $t$ in general for the family of potentials defined by $V(\phi)=V_{0} \phi^{n} \exp \left(-\lambda \phi^{m}\right)$. In [16] a perturbative analysis was employed at large $t$ to arrive at the constraint $m \leq 1$ necessary for inflation at late times (corresponding to large $\phi$ for the particular parameter ranges considered). A similar treatment here at small times would be innapropriate because of the ambiguities concerning the definition of "early-time" encountered in section 3.2. Instead we shall treat the scalar field as a time variable, as outlined in [19]. The monotonicity of $\dot{\phi}$ that is required to do this guarantees that each extremum of $t$ will correspond to a unique extremum of $\phi$ and vice-versa; examining the system at large and small $\phi$ is sufficient to obtain the complete solution at early and late times. We thus complete our picture of inflationary models arising from Eq. (1.1) by analysing their behaviour at small $\phi$.

Conditions on the potential such that inflation can occur, derived from perturbation theory within this $\phi$-parameterised formalism concur with those obtained from the PSRA 2. We shall be concerned in particular with $\epsilon_{V}$ since it offers a good approximation to $\epsilon_{H}$,

\footnotetext{
${ }^{2}$ The applicability of this scheme at small $t$ could be regarded as questionable, since the PSRA only applies generically once the system has had sufficient time to settle into the inflationary attractor [9, 17] . Here, we assume that the slow-rolling portion of the potential is large enough to allow the attractor to be
} 
the smallness of which is linked directly to the positivity of $\ddot{a}$. Inspecting the form of $\epsilon_{V}$ for these models, given in Eq. (2.12) we see immediately that for small $\phi, \epsilon_{V}$ will always blow up unless $n=0 ; n=0$ is thus a necessary condition for inflation at small $\phi$. In this case Eq. (2.12) simplifies considerably to become

$$
\epsilon_{V}=\frac{1}{2} \lambda^{2} m^{2} \phi^{2 m-2}
$$

Requiring this to be small as $\phi \rightarrow 0$ is equivalent to demanding that $2 m-2$ be positive or zero, leading to the constraint $m \geq 1$ for inflation. The bound in parameter space at small $\phi$ is thus opposite to the bound at large $\phi$.

Next we look for small $\phi$ solutions. Differentiating $V(\phi)$ and substituting into Eq. (2.9) yields

$$
\dot{\phi}=\sqrt{\frac{V_{0}}{3}} \lambda m \phi^{m-1} \exp \left(-\frac{\lambda}{2} \phi^{m}\right) .
$$

The choice $m=1$ leads to power-law inflation, and so we shall be concerned only with $m>1$. Two distinct cases arise:

\subsection{The case $\mathbf{m}>1, \neq 2$}

The analysis in this general case for positive $m$ at small $\phi$ parallels that of section 3.2 .2 for negative $m$ at large $\phi$ and we obtain the approximate solution already given in Eqs. (3.21) - (3.24). When $1<m<2, t \rightarrow 0$ as $\phi \rightarrow 0$. If $m>2, t \rightarrow-\infty$ as $\phi \rightarrow 0$ and the solution is valid at early times. Also, $\gamma_{2}(t)$ tends to unity in this limit, as expected.

attained well before leaving the early-time asymptopia, although in practice this should be checked. 


\subsection{The case $\mathrm{m}=2$}

Here we discover a new type of behaviour. Eq. (4.2) integrates approximately at small $\phi$, giving

$$
t(\phi)=\sqrt{\frac{3}{V_{0}}} \frac{1}{2 \lambda}(\ln \phi) \exp \left(-\frac{\lambda}{2} \phi^{2}\right) .
$$

The full time-evolution, valid at small $\phi$, is then given by

$$
\begin{aligned}
\phi(t) & =\exp \left[2 \sqrt{\frac{V_{0}}{3}} \lambda t\right], \\
H(t) & =\sqrt{\frac{V_{0}}{3}} \exp \left[-\frac{\lambda}{2} \exp \left(4 \sqrt{\frac{V_{0}}{3}} \lambda t\right)\right], \\
a(t) & \propto \exp \left[\sqrt{\frac{V_{0}}{3}} t \gamma_{4}(t)\right],
\end{aligned}
$$

with,

$$
\gamma_{4}(t) \equiv \exp \left[-\frac{\lambda}{2} \exp \left(4 \sqrt{\frac{V_{0}}{3}} \lambda t\right)\right] .
$$

Eq. (4.3) reveals that $t \rightarrow-\infty$ as $\phi \rightarrow 0$ and $\gamma_{4}(t) \rightarrow 1$.

Models with $m<1$ inflate at large $t$, whereas those with $m=1$ inflate for all $t$ (powerlaw inflation). It should not be surprising then that the solutions inflate at small $t$ when $m>1$. Both the solutions (3.24) and (4.6), presented above, tend to de Sitter expansion in the small $t$ limit.

\section{Density and Gravitational Wave Perturbations}

A period of inflation in the early universe provides a means to generate the small fluctuations from which the large-scale structure we observe in the universe today can have grown. The stretching and freezing-out of quantum excitations in the inflaton and graviton fields during inflation gives rise to a spectrum of scalar and tensor fluctuations in the cosmic microwave 
background; they can be classified by the spectral indices $\hat{n}_{s}$ and $\hat{n}_{g}$ respectively [3]. These are defined as

$$
\begin{aligned}
\hat{n}_{s}-1 & =\frac{d \ln \delta_{H}^{2}(k)}{d \ln k}, \\
\hat{n}_{g} & =\frac{d \ln \sigma_{H}^{2}(k)}{d \ln k},
\end{aligned}
$$

where

$$
\delta_{H}(k)=\frac{\delta \rho}{\rho}
$$

is the scalar density contrast on a scale corresponding to comoving wavenumber $k=a H$ ( $a H$ evaluated when the fluctuation crossed outside the Hubble radius during inflation) and $\sigma_{H}(k)$ is the dimensionless strain induced by the gravitational wave perturbations on scale $k$. During slow-rolling inflation, one may express $\hat{n}_{s}$ and $\hat{n}_{g}$ to first order in the PSRA parameters as [3]

$$
\begin{aligned}
1-\hat{n}_{s} & =6 \epsilon_{V}-2 \eta_{V}, \\
\hat{n}_{g} & =-2 \epsilon_{V} .
\end{aligned}
$$

Furthermore, the ratio of the amplitudes of tensor to scalar modes in the COBE signal on a scale corresponding to the $l^{\text {th }}$ multipole of the temperature anisotropy expansion is

$$
R_{l}=\frac{25}{2} \epsilon_{V}
$$

where $l$ is the multipole corresponding to the scale $k(\phi)$ at which $\epsilon_{V}$ is evaluated. Tensor fluctuations are thus always sub-dominant in slow-roll and $\hat{n}_{s}$ is close to unity, as observed by COBE 4 .

Much work has been carried out recently using the second-order results of [20]. However here the second-order corrections to Eqs. (5.4) - (5.6) prove too cumbersome to be of use, as well as exceeding the accuracy of the approximate results already presented. Confining 
ourselves to a first-order treatment and utilising Eqs. (2.12) and (2.13) yields

$$
\begin{aligned}
1-\hat{n}_{s} & =\frac{2}{\phi^{2}}\left[n\left(\frac{n}{2}+1\right)+\lambda m(m-n-1) \phi^{m}+\frac{\lambda^{2} m^{2}}{2} \phi^{2 m}\right] \\
\hat{n}_{g} & =-\frac{1}{\phi^{2}}\left(n-\lambda m \phi^{m}\right)^{2} \\
R_{l} & =\frac{25}{4 \phi^{2}}\left(n-\lambda m \phi^{m}\right)^{2},
\end{aligned}
$$

These expressions are evaluated at horizon crossing when $k(\phi)=a H$. The relation between the field $\phi$ and scale $k$ is established by considering the number of e-foldings of contraction experienced by the comoving Hubble length $(a H)^{-1}$, given by

$$
\bar{N}(k) \equiv \ln \frac{(a H)_{f}}{(a H)_{i}}
$$

where the subscripts $i$ and $f$ denote the initial and final values of $(a H)$. The functional form of $\bar{N}(k)$ is irrelevant for our discussion here although an expression is given in 17]. More pertinent here is the counterpart function, $\bar{N}(\phi)$, given to lowest order in PSRA parameters by 17

$$
\bar{N}\left(\phi_{1}, \phi_{2}\right)=-\sqrt{\frac{4 \pi}{M_{P l}^{2}}} \int_{\phi_{1}}^{\phi_{2}} \frac{1}{\sqrt{\epsilon_{V}(\phi)}} d \phi .
$$

With a view to numerical evauation, the $M_{P l}$ factors have been restored to this expression and are retained hereafter. The form of $\bar{N}$ is dependent upon the range of $m$ and $n$ and the appropriate limit of $\phi$ required to obtain inflationary behaviour:

(i) $0<m<1$ and $m \leq 0, n=0$ as $\phi \rightarrow \infty$ or $m>1$ as $\phi \rightarrow 0$. In these cases we obtain,

$$
\bar{N}\left(\phi_{1}, \phi_{2}\right) \rightarrow \frac{1}{\lambda m(2-m)} \frac{8 \pi}{M_{P l}^{2}}\left[\phi_{2}^{2-m}-\phi_{1}^{2-m}\right]
$$

(ii) $m \leq 0, n \neq 0, \phi \rightarrow \infty$. The result here is simpler,

$$
\bar{N}\left(\phi_{1}, \phi_{2}\right) \rightarrow \frac{1}{n} \frac{8 \pi}{M_{P l}^{2}}\left[\phi_{1}^{2}-\phi_{2}^{2}\right]
$$


Eqs. (5.12) and (5.13), together with $\bar{N}(k)$ establish the $\phi-k$ correspondences necessary to relate quantum fluctuations at a "time" $\phi$ to microwave background fluctuations on a scale $k$.

A successful model of inflation requires a minimum of 70 e-foldings of comoving contraction (to solve the horizon and flatness problems) before the conditions $\epsilon_{V}, \eta_{V} \ll 1$ become violated [6]. This constraint provides bounds on the potential in models where $\phi_{1}$ is fixed by particular initial conditions. When the potential possesses a minimum this does not apply; then, $\epsilon_{V}, \eta_{V} \rightarrow 0$ at late times, and such models must be modified if inflation is to end. Potentially, this permits an enormous amount of expansion.

It was seen earlier that the amplitude of the fluctuations arising from slow-rolling inflation is dominated by the scalar component. This has been calculated to zeroth order in the PSRA [3] as

$$
\delta_{H}^{2}=\frac{512 \pi}{75 M_{P l}^{6}} \frac{V^{3}}{V^{\prime 2}}
$$

For the potential of Eq. (1.1) this implies

$$
\delta_{H} \simeq \frac{16 \sqrt{2}}{5 M_{P l}^{3}} \sqrt{\frac{V_{0}}{3}} \frac{\phi^{\frac{n}{2}+1} \exp \left(-\frac{\lambda}{2} \phi^{m}\right)}{\left|n-\lambda m \phi^{m}\right|} .
$$

The form of $\bar{N}(k)$ given in 17] implies that $(a H)^{-1}$ shrunk to scales of astrophysical interest at approximately $\phi=\phi_{*}, 60$ comoving e-folds before the end of inflation, a figure which is weakly sensitive to the physics of reheating. Models in which inflation ends naturally (namely those occupying the regions $m<0, n \geq 0$ or $m>1, n=0$ of $m$ - n parameter space) can thus be constrained further by demanding $\delta_{H}\left(k_{*}\right)$ be consistent with observational data. This requires knowledge of $\phi\left(\epsilon_{V}\right)$ so that one may obtain a reliable approximation to $\phi_{\text {end }} \simeq \phi\left(\epsilon_{V}=1\right)$, the value of $\phi$ at which inflation ends. In particular, if 
$n=0$ we find

$$
\phi_{\text {end }}=\left(\frac{16 \pi}{\lambda^{2} m^{2} M_{P l}^{2}}\right)^{\frac{1}{2 m-2}} .
$$

Which, in conjunction with Eq. (5.12) yields

$$
\phi_{*}^{2-m}=\left(\frac{16 \pi}{\lambda^{2} m^{2} M_{P l}^{2}}\right)^{\frac{2-m}{2 m-2}}+\frac{15 \lambda m M_{P l}^{2}}{2 \pi}(m-2) .
$$

Substituting this result, and $n=0$, into Eq. (5.15) we obtain the model prediction,

$$
\left[\frac{5 \lambda m M_{P l}^{3}}{16 \sqrt{2}} \sqrt{\frac{3}{V_{0}}} \delta_{H}\right]^{\frac{2-m}{1-m}} \approx\left(\frac{16 \pi}{\lambda^{2} m^{2} M_{P l}^{2}}\right)^{\frac{2-m}{2 m-2}}+\frac{15 \lambda m M_{P l}^{2}}{2 \pi}(m-2) .
$$

Under particular circumstances this expression simplifies. When $m=2$,

$$
\delta_{H} \approx \frac{4}{5 M_{P l}^{2}} \sqrt{\frac{2 V_{0}}{3 \pi}}
$$

and as $m \rightarrow \pm \infty$,

$$
\delta_{H} \rightarrow \frac{16}{5 \lambda m M_{P l}^{3}} \sqrt{\frac{2 V_{0}}{3}}\left[\frac{15}{2 \pi} \lambda m^{2} M_{P l}^{2}\right]^{\frac{1-m}{2-m}} .
$$

The most recent analysis of the COBE data [5] gives $\delta_{H} \simeq 2.3 \times 10^{-5}$ which, once $\lambda$ and $m$ have been fixed, imposes a constraint on the value of $V_{0}$.

\section{Conclusions}

We have studied the behaviour of inflationary universe models emerging when one posits a form for the inflaton potential containing both exponential and power-law factors. By examining the validity of the slow-roll approximation in these models at the extremes of $\phi$ we have determined the structure of the $m-n$ parameter space at early and late times. We have isolated the regions in which inflation may occur in these two eras and we have obtained a wide variety of qualitatively different solutions to the equations of motion, dependent to a large extent on the value of $m$. We find that when $m>1$, quasi-de Sitter inflation is manifest 
at early-times provided $n=0$ but this is not a feature as $t \rightarrow \infty$, where the potential tends to zero faster than the kinetic energy of the field, and all models are non-inflationary. This is the case at all times when $m>1, n \neq 0$; these models can never inflate. Within the broad range $0 \leq m \leq 1$ inflation is generic at late times for all $n$. In particular, when $m=0$, the solutions exhibit polynomial-chaotic or intermediate inflationary behaviour (dependent upon the sign of $n$ ) and, if $n=0$, we recover the de Sitter solution. If $m=1$ and $n=0$ we have power-law inflation; when $n \neq 0$ the behaviour will asymptote to the power-law form at large $t$. When $0<m<1$ the scale factor approaches a new type of behaviour, proportional to $\exp \left(\ln ^{(2-m) / m} t\right)$ as $t \rightarrow \infty$. If $m<0$ and $n \geq 0$, inflation occurs at early times; but, if $n<0$, it proceeds once more as a late-time phenomenon and the precise form of the inflationary behaviour is determined by the specific value of $n$.

In summary, our analysis extends the treatment presented in [16] to establish a set of sufficient conditions for inflation to occur at early or late epochs, summarised in Table 1 below.

\begin{tabular}{||c|c|c||}
\hline $\begin{array}{c}\text { Early } \\
\text { Times }\end{array}$ & Late & All \\
Times & Times \\
\hline \hline$m \leq 0, n \geq 0$ & $0 \leq m \leq 1$ & $m=1, n=0$ \\
or & or & or \\
$m \geq 1, n=0$ & $m \leq 0, n<0$ & $m=n=0$ \\
\hline
\end{tabular}

Table 1: The conditions on $m$ and $n$ in $V(\phi)=V_{0} \phi^{n} \exp \left(-\lambda \phi^{m}\right)$ for inflation to occur.

The calculation of the perturbation spectra produced in these theories allows further constraints to be placed on $m, n$ and $\lambda$ by comparison with microwave background observation. Moreover, the restrictions imposed on $V_{0}$ from the amplitude of scalar fluctuations 
go some way toward determining the energy scale at which inflation occurs [21].

\section{Acknowledgements}

PP is supported by a PPARC Postgraduate Studentship and JDB by a PPARC Senior Fellowship.

\section{References}

[1] E. W. Kolb and M. S. Turner, The Early Universe, Addison-Wesley, (1990).

[2] A. D. Linde, Particle Physics and Inflationary Cosmology, Gordon and Breach, (1990).

[3] A. R. Liddle and D. H. Lyth, Phys. Rep. 231, 1 (1993).

[4] G. F. Smoot et al, Astrophys. J. Lett. 396, L1 (1992).

[5] K. Górski et al, Astrophys. J. Lett. 430, L89 (1994).

[6] A. H. Guth, Phys. Rev. D23, 347 (1981).

[7] J. J. Halliwell, Phys. Lett. B185, 341 (1987).

[8] A. B. Burd and J. D. Barrow, Nucl. Phys. B308, 929 (1988).

[9] D. S. Salopek and J. R. Bond, Phys. Rev. D42, 3936 (1990).

[10] A. D. Linde, Phys. Lett. B129, 177 (1983).

[11] F. Lucchin and S. Matarrese, Phys. Rev. D32, 1316 (1985).

[12] A. G. Muslimov, Class. Quant. Grav 7, 231 (1990).

[13] J. D. Barrow, Phys. Lett. B235, 40 (1990). 
[14] J. D. Barrow and P. Saich, Phys. Lett. 249, 406 (1990).

[15] J. D. Barrow and A. R. Liddle, Phys. Rev. D47, R5129 (1993).

[16] J. D. Barrow, Phys. Rev. D48, 1585 (1993).

[17] A. R. Liddle, P. Parsons and J. D. Barrow, Phys. Rev. D50, 7222 (1994).

[18] F. W. J. Olver, Asymptotics and Special Functions, Academic Press, (1974).

[19] J. E. Lidsey, Phys. Lett. B273, 42 (1991).

[20] E. D. Stewart and D. H. Lyth, Phys. Lett B302, 171 (1993).

[21] A. R. Liddle, Phys. Rev. D49, 739 (1994). 